\title{
Potential analysis of multidisciplinary biopsychosocial rehabilitation for patients with chronic low back pain Potentialanalyse der multidisziplinären biopsychosozialen Rehabilitation für Patienten/-innen mit chronischen Rückenschmerzen
}

\author{
Beate Schüßler ${ }^{1 *}$, Angsar Abel', Milena Holzer', \\ Ruth Meise' ${ }^{1}$, Sebastian Voigt-Radloff², Axel Schäfer ${ }^{3}$ \\ ${ }^{1}$ Fakultät Soziale Arbeit und Gesundheit, HAWK Hildesheim, \\ Hildesheim, GERMANY \\ *bat.schuessler@gmail.com \\ ${ }^{2}$ Cochrane Deutschland, Universitätsklinikum Freiburg, \\ Freiburg, GERMANY \\ ${ }^{3}$ Hochschule Bremen, Fakultät Gesellschaftswissenschaften, \\ Studiengang Angewandte Therapiewissenschaften
} Logopädie und Physiotherapie, Bremen, GERMANY

Received 2 October 2015, accepted 21 November 2016, available online 12 February 2017

\begin{abstract}
Health problem: The prevalence of low back pain ranges from 74 to $85 \%$, almost everybody in Germany suffers from low back pain once in his or her life. The recurrence rate within twelve months is $62 \%$, which often leads to inability to work and reduction in earning capacity. The rate of chronic manifestation of low back pain is $5 \%$. Subsequent loss of mobility and functional disability lowers quality of life and participation in private life and at work.

Evidence: A recent Cochrane review (Kamper et al., 2014) analysed six (outcome disability) and seven (outcome pain) studies with 722 and 821 participants respectively and showed that multidisciplinary rehabilitation compared to usual care has a small, but clinically relevant effect (pain: SMD $-0,21$ [CI 95\% $-0,37 ;-0,04]$; disability: SMD $-0,23$ [CI 95\% $-0,4 ;-0,06]$ ). There is a lack of evidence about the intervention conception, patient-centred outcomes and the cost effectiveness.

Implication for research: Prior to a randomised controlled trial a feasibility study about individualised multidisciplinary biopsychosocial rehabilitation compared to usual care considering people with chronic low back pain at employable age in Germany is needed. The applicability of available manuals, patient-centred outcomes and cost effectiveness should be explored.
\end{abstract}

\begin{abstract}
Gesundheitsproblem: Rückenschmerz hat eine Lebenszeitprävalenz von 74-85 \%, sodass fast jeder Mensch in Deutschland einmal von einer Rückenschmerzepisode betroffen sein wird. $62 \%$ der Betroffenen bekommen innerhalb von zwölf Monaten nach der ersten Rückenschmerzepisode ein Rezidiv, was häufig zu Arbeitsunfähigkeit oder langfristig zu Erwerbsminderung führen kann. Die Chronifizierungsrate von Rückenschmerzen liegt bei 5-8 \%. Durch Mobilitätsverlust und Funktionseinschränkung kann es zu einem Verlust an Lebensqualität sowie zu Einschränkungen der Partizipation im privaten sowie beruflichen Bereich kommen.

Evidenzlage: Ein Cochrane Review von Kamper et al. (2014) zeigt auf Grundlage von sechs Studien für den Endpunkt ,funktionelle Beeinträchtigung“ bzw. sieben Studien für den Endpunkt „Schmerz“ mit 722 bzw. 821 Teilnehmenden, dass multidisziplinäre Rehabilitation im Vergleich zu „normaler“ Versorgung hinsichtlich funktioneller Beeinträchtigung und Schmerz einen kleinen, jedoch klinisch relevanten Effekt zeigt (Schmerz: SMD -0,21 [CI $95 \%-0,37 ;-0,04]$; Beeinträchtigung SMD -0,23 [Cl 95 \% -0,4; -0,06]). Es besteht eine Evidenzlücke in Bezug auf die Konzeption der Interventionen, die klienten/-innenrelevanten Endpunkte sowie die Kosten-Nutzen-Effektivität.

Forschungsempfehlung: Es empfiehlt sich als Vorbereitung einer Wirksamkeitsstudie eine Machbarkeitsstudie zu individualisierter multidisziplinärer biopsychosozialer Rehabilitation im Vergleich zur Routineversorgung bei Patienten/-innen mit chronischem Rückenschmerz im erwerbsfähigen Alter in Deutschland. Es gilt, die Anwendbarkeit vorhandener Manuale, klienten/-innenzentrierte Endpunkte und die Kosten-Nutzen-Effektivität zu prüfen.
\end{abstract}

\section{Keywords}

Potential analyse - chronic low back pain - multidisciplinary biopsychosocial rehabilitation

\section{Keywords}

Potentialanalyse - Chronische Rückenschmerzen - Multidisziplinäre biopsychosoziale Rehabilitation 


\section{EINLEITUNG ZUM FORMAT DER POTENTIALANALYSE}

Dieser Artikel im Format einer Potentialanalyse (S. Voigt-Radloff, Lang \& Antes, 2014) hat das Ziel, die Übertragung der Intervention „,multidisziplinäre biopsychosoziale Rehabilitation“ (MBR) für Patienten/innen mit chronischen Rückenschmerzen in den deutschsprachigen Zielkontext zu prüfen und eine Forschungsempfehlung abzuleiten.

Generell sollen im Rahmen von Potentialanalysen vielversprechende Interventionen für versorgungsrelevante Gesundheitsprobleme ausfindig gemacht werden und deren Transfer in die Routineversorgung unterstützt werden. Vielversprechend ist eine Intervention dann, „wenn sie nachweislich wirksam und in die Versorgung übertragbar ist und wenn es wahrscheinlich ist, dass die Umsetzung in der Routine einen wesentlichen Beitrag zur Lösung des Gesundheitsproblems leisten kann“ (S. Voigt-Radloff et al., 2016, S. 13).

Nach dem Leitfaden „Forschung zu komplexen Interventionen in der Pflege- und Hebammenwissenschaft und in den Wissenschaften der Physiotherapie, Ergotherapie und Logopädie“ (S. Voigt-Radloff et al., 2016) ist es dazu nötig, auf Grundlage einer systematischen Literaturrecherche Gesundheitsprobleme und Folgen für die Betroffenen zu beschreiben, die Endpunkte, für welche die meisten Betroffenen Verbesserungen wünschen, zu benennen, die Folgen für die Gesellschaft $\mathrm{zu}$ beschreiben und $\mathrm{zu}$ beziffern sowie letztlich die Versorgung durch Gesundheitsfachberufe in Art und Häufigkeit darzustellen. Darauf folgt eine Beschreibung der Methode der Literaturrecherche und Bewertung der gefundenen Evidenz. Die Recherche nach Evidenz erfolgt nach einer an dem PICOS Schema ausgerichteten Fragestellung und soll zunächst das aktuellste Cochrane Review dazu berücksichtigen, ob bei Fehlen eines solchen auch andere qualitätsgesicherte Reviews sowie danach publizierte Primärstudien berücksichtigt werden können (S. Voigt-Radloff et al., 2014).

Entsprechend wird die Evidenzlage der Intervention inklusive potentieller Wirkmechanismen endpunktspezifisch beschrieben, die Darstellung wird mit einer Tabelle zur Wirksamkeit und Evidenzqualität (GRADE Evidenzprofil) (Langer et al., 2012) unterstützt. Weiterhin gibt es eine Tabelle zu Evidenzlücken, in der alle nach dem systematischen Review veröffentlichten Studien aufgelistet werden. Die Ergebnisse dieser Studien werden nicht berichtet. Dies soll Fehlinterpretationen vermeiden, die auftreten können, wenn Ergebnisse ohne Prüfung der Evidenzqualität, Heterogenität und Gewichtung rein narrativ dargestellt werden (Murad et al., 2014).

Daraus resultierend wird am Ende der Potentialanalyse eine begründete Empfehlung zur weiteren Forschung in
Bezug aufMachbarkeit, Wirksamkeitoder Implementation der Intervention im Zielkontext formuliert oder ggf. von weiteren Untersuchungen abgeraten, wenn das Potential der Intervention nicht ausreichend erscheint (S. VoigtRadloff et al., 2016).

Der methodische Leitfaden für Potentialanalysen ist im Internet unter https://freidok.uni-freiburg.de/data/10702 frei verfügbar.

\section{DAS GESUNDHEITSPROBLEM}

\section{Das Gesundheitsproblem und die Folgen für Betroffene}

Rückenschmerz ist eines der häufigsten und kostenintensivsten Gesundheitsprobleme weltweit mit gravierenden Folgen für die Lebensqualität der Betroffenen (Duthey, 2013). Rückenschmerz hat eine Lebenszeitprävalenzvon 74-85\%, sodassfastjederMensch in Deutschland einmal von einer Rückenschmerzepisode betroffen sein wird (RKI, 2012, S. 13). Dabei sind Frauen häufiger betroffen als Männer (BÄK, KBV \& AWMF 2010, S. 42) und die Prävalenz verläuft annähernd linear zum Alter (RKI, 2012, S. 13; BÄK et al., 2010, S. 42). Bei einer Querschnittsuntersuchung zwischen 2003 und 2006 wurde ein Zusammenhang von niedrigem Bildungsniveau zur Erkrankungshäufigkeit festgestellt (BÄK et al., 2010, S. 42).

Rückenschmerz liegt auf Platz drei der zwanzig häufigsten HauptdiagnosenvollstationärbehandelterPatienten/-innen in Vorsorge- und Rehabilitationseinrichtungen und ist die häufigste Ursache für Arbeitsunfähigkeit (Statistisches Bundesamt, 2012, o. S.). Auch wenn ein Großteil der Betroffenen nach wenigen Wochen beschwerdefrei ist, bekommen $62 \%$ der Betroffenen innerhalb von zwölf Monaten nach der ersten Rückenschmerzepisode ein Rezidiv (RKI, 2012, S. 12), was häufig zu Arbeitsunfähigkeit (11,7 AU-Tage pro Fall) oder langfristig zu Erwerbsminderung führen kann. $80 \%$ aller Rückenschmerzen sind unspezifische Rückenschmerzen (RKI, 2012, S. 10), d.h. eine strukturelle Ursache ist hier nicht nachweisbar.

Als Risikofaktoren für eine Chronifizierung gelten arbeitsbezogene psychosoziale Faktoren wie Unzufriedenheit und Monotonie am Arbeitsplatz sowie psychische Faktoren, wie depressive Episoden und Katastrophisieren (Waddell, 2004). Weitere prädisponierende Faktoren sind das Krankheitsverhalten, wie zu lange Bettruhe und Passivität sowie ein niedriger sozialer Status und ein niedriges Bildungsniveau. Die genannten Variablen zeigen die Komplexität des Gesundheitsproblems. (RKI, 2012, S. 8f). Die Rate der Chronifizierung von Rückenschmerzen liegt bei 5-8 \% (Kohlmann, 2003, S. 327f.). 
Tabelle 1: GRADE Evidenz Profil „MBR versus Usual Care bei Patienten mit chronischen Rückenschmerzen“

\begin{tabular}{|c|c|c|c|c|c|c|c|c|c|c|c|}
\hline \multicolumn{6}{|c|}{ Bewertung der Qualität } & \multicolumn{6}{|c|}{ Übersicht der Ergebnisse } \\
\hline & & & & & & \multicolumn{2}{|c|}{ Anzahl Patienten } & \multirow[b]{2}{*}{$\begin{array}{l}\text { Std. } \\
\text { Mean } \\
\text { Diffe- } \\
\text { rence }\end{array}$} & \multirow[b]{2}{*}{$\begin{array}{c}\mathrm{Cl} \\
95 \%\end{array}$} & \multirow[b]{2}{*}{ Effekt } & \multirow[b]{2}{*}{ Qualität } \\
\hline $\begin{array}{l}\text { Anzahl der } \\
\text { Studien }\end{array}$ & $\begin{array}{l}\text { Limita- } \\
\text { tionen }\end{array}$ & $\begin{array}{l}\text { Inkonsis- } \\
\text { tenz }\end{array}$ & $\begin{array}{l}\text { Indirekt- } \\
\text { heit }\end{array}$ & $\begin{array}{l}\text { Inpräzi- } \\
\text { sion }\end{array}$ & $\begin{array}{l}\text { Publika- } \\
\text { tions-Bias }\end{array}$ & MBR & $\begin{array}{l}\text { Usual } \\
\text { Care }\end{array}$ & & & & \\
\hline \multicolumn{12}{|c|}{ Disability Longterm } \\
\hline \multicolumn{12}{|c|}{ Pain Longterm } \\
\hline $7 \mathrm{RCT}^{* *}$ & $\begin{array}{c}\text { Schwer- } \\
\text { wiegende } \\
\text { Limitatio- } \\
\text { nen }\end{array}$ & $\begin{array}{l}\text { Keine } \\
\text { schwer- } \\
\text { wiegende } \\
\text { Inkonsis- } \\
\text { tenz }\end{array}$ & $\begin{array}{c}\text { Keine } \\
\text { schwer- } \\
\text { wiegende } \\
\text { Indirekt- } \\
\text { heit }\end{array}$ & $\begin{array}{l}\text { Keine } \\
\text { schwer- } \\
\text { wiegende } \\
\text { Inpräzi- } \\
\text { sion }\end{array}$ & unerkannt & $448 / 821$ & $373 / 821$ & $-0,21$ & $\begin{array}{l}-0,37 \\
-0,04\end{array}$ & $\begin{array}{l}\text { Kleiner } \\
\text { Effekt }\end{array}$ & moderat \\
\hline
\end{tabular}

Abkürzungen: RCT-Randomised Controlled Trial; Std.-Standard; Cl-Confidence Interval; MBR-Multidisziplinäre biopsychosoziale Rehabilitation * Bendix et al. (1996); Lambeek et al. (2010); Linton et al. (2005); Lukinmaa (1989); Strand et al. (2001); Von Korff et al. (2005)

** Bendix et al. (1996); Bendix et al. (1998); Lambeek et al. (2010); Linton et al. (2005); Lukinmaa (1989); Strand et al. (2005); Von Korff et al. (2005)

Tabelle 2: Evidenzlücken

\begin{tabular}{|c|c|}
\hline \multirow{3}{*}{$\begin{array}{l}\text { Nachfolgende Studien } \\
\text { ohne Ergebnisbewertung }\end{array}$} & $\begin{array}{l}\text { Es konnten drei nachfolgende Studien zu MBR bei CLBP identifiziert werden: } \\
\text { Monticone et al. (2014) untersuchten den Effekt von MBR gegenüber Usual Care bei Patienten mit CLBP nach } \\
\text { acht Wochen und drei Monaten. Endpunkte: Kinesiophobie, Katastrophisierung, funktionelle Beeinträchtigung, } \\
\text { Lebensqualität und Gangbild. }\end{array}$ \\
\hline & $\begin{array}{l}\text { Pieber et al. (2014) untersuchten die Kurz- und Langzeiteffekte von MBR nach } 6 \text { und } 18 \text { Monaten bei Patienten mit } \\
\text { CLBP. Endpunkte: Schmerz, Kraft, Funktion und Lebensqualität. }\end{array}$ \\
\hline & $\begin{array}{l}\text { Tavafian, Jamshidi, Mohammad (2014) untersuchten die Wirksamkeit der Intervention „MBR“ im Vergleich zur } \\
\text { Kontrollgruppe (Medikamente) langfristig (12 Monate) hinsichtlich Lebensqualität und funktioneller Beeinträchtigung. }\end{array}$ \\
\hline \multirow{4}{*}{$\begin{array}{l}\text { Klientenrelevante } \\
\text { Endpunkte }\end{array}$} & Kamper et al. (2014) gehen nicht auf klientenzentrierte Endpunkte ein. \\
\hline & $\begin{array}{l}\text { Bunzli et al. (2013) untersuchten die Erfahrungen von Patienten mit CLBP und zeigten, dass drei } \\
\text { Hauptproblematiken thematisiert werden: Zum einen wird die negative Beeinflussung des sozialen Konstrukts und } \\
\text { des täglichen Lebens genannt, sodass keine Weiterentwicklung und Zukunft möglich zu sein scheint. Als zweites } \\
\text { stehen die psychosozialen Auswirkungen von CLBP im Vordergrund und als drittes der Umgang mit CLBP. Darunter } \\
\text { fallen der eigene Umgang mit den Schmerzen durch Vermeidungsverhalten und der Umgang der Umwelt mit dem } \\
\text { chronischen Schmerzpatienten, welcher die Legitimation der Schmerzen häufig in Frage stellt. }\end{array}$ \\
\hline & $\begin{array}{l}\text { Eaves et al. (2015) interviewten Patienten mit CLBP über ihre Erwartungen an eine Therapie. Die Patienten } \\
\text { erwarten von einer Therapie eine Erklärung für ihr Problem, um eine Legitimation gegenüber sich und der } \\
\text { Umwelt für ihr Krankheitsbild zu erhalten Sie beschreiben den Schmerz als omnipräsent, sodass Arbeit und } \\
\text { Alltag eingeschränkt werden und Zukunftspläne nicht möglich scheinen. Es bestehen Ängste vor Jobverlust und } \\
\text { finanziellen Schwierigkeiten. Sie wünschen sich eine Unterstützung bei dem Umgang mit den Schmerzen, um die } \\
\text { Stimmung zu verbessern Bewältigungsstrategien zu erlernen. }\end{array}$ \\
\hline & $\begin{array}{c}\text { Trotz dieser Studien besteht eine große Lücke hinsichtlich der klientenrelevanten Endpunkte bei CLBP. Es ist } \\
\text { notwendig weiter gezielte Forschung in diese Richtung zu betreiben. }\end{array}$ \\
\hline Kosten & $\begin{array}{l}\text { Das Cochrane Review berichtet über eine Forschungslücke des Kosten-Nutzen-Effekts von MBR. Es wird in } 15 \\
\text { Studien über circa } 100 \text { Kontaktstunden berichtet, die für die Intervention „MBR“ benötigt werden. Die Kosten von } \\
\text { „Usual Care“ müssen gegenüber gestellt werden. Zusätzlich müssen Kosten für Arbeitsausfälle bedacht werden. }\end{array}$ \\
\hline Langzeitwirkungen & $\begin{array}{c}\text { Kamper et al. (2014) berichten von Langzeitwirkungen (12 Monate oder länger) hinsichtlich funktioneller } \\
\text { Beeinträchtigung, Schmerzen und Arbeitsfähigkeit. Bei funktioneller Beeinträchtigung und Schmerzen zeigte sich } \\
\text { ein kleiner Effekt, welcher allerdings eine klinische Relevanz haben könnte. Bei Arbeitsfähigkeit konnten keine } \\
\text { signifikanten oder klinisch relevanten Effekte gefunden werden. }\end{array}$ \\
\hline Unerwünschte Effekte & Kamper et al. (2014) berichten nicht von unerwünschten Effekten der Intervention „MBR“ \\
\hline
\end{tabular}

Abkürzungen: MBR-Multidisziplinäre biopsychosoziale Rehabilitation; CLBP-Chronische Rückenschmerzen 
Wird der Verlust an gesunden Lebensjahren durch Rückenschmerzen betrachtet, dann rangiert chronischer Rückenschmerz bei Männern auf Rang zwei und bei Frauen auf Rang eins (Plass et al., 2014, S. 636). Durch den Mobilitätsverlust und die Funktionseinschränkungen kann es zu einer allgemeinen Dekonditionierung und einem Verlust an Lebensqualität sowie zu Einschränkungen der Partizipation im privaten sowie beruflichen Bereich kommen (RKI, 2012, S. 11).

\section{Klienten/-innenzentrierte Endpunkte}

Ein Kritikpunkt an vielen Studien ist es, dass Endpunkte gewählt werden, die aus Sicht der Betroffenen wenig bedeutsam sind. Im Folgenden wird daher die Frage beleuchtet, welche Endpunkte für Betroffene bedeutsam und relevant sind. Ein primärer Endpunkt in vielen Studien zu chronischem Rückenschmerz ist etwa die Schmerzintensität. Als gängige Instrumente werden die Visuelle Analogskala (VAS) oder die Numerische Analogskala (NRS) verwendet, da sie valide und gut vergleichbar sind (Kamper et al., 2014, S.6ff.). Da Schmerzen für die Betroffenen oft das Hauptproblem sind, ist eine Bewertung, die über die reine Messung der Schmerzintensität hinausgeht, zu empfehlen. Eine grundlegende Ausweitung der Schmerzbeurteilung in Hinblick auf Qualität, Dauer und daraus resultierender Beeinträchtigung wurde bereits vor längerer Zeit vorgeschlagen (Borkan, Reis, Hermoni \& Biderman, 1995, S. 977ff.; Bunzli, Watkins, Smith, Schütze \& O’Sullivan, 2013, S. 14). Es gilt, hierfür valide Instrumente $\mathrm{zu}$ verwenden, um einer besseren Darstellung des Schmerzerlebens gerecht zu werden.

Ferner sollten im Rahmen eines biopsychosozialen Ansatzes patienten/-innenzentrierte Endpunkte für Aktivität, Partizipation und gesundheitsbezogene Lebensqualität ausgewählt werden (Ferrans, Zerwic, Wilbur \& Larson, 2005). So wurden bereits mittels des Canadian Occupational Performance Measure (COPM) aus der Ergotherapie patientenzentrierte Endpunkte bei Patienten/-innen mit Rückenschmerzen gemessen (Walsh, Kelly, Johnson, Shanmugasundram \& Bennetts, 2003, S.87ff.). Die Ergebnisse dieser Studie zeigten, dass die Einschränkungen beim Gehen (ca. $56 \%$ ), starke Schmerzepisoden (ca. $39 \%$ ) und das Sitzen (ca. $31 \%$ ) Probleme waren, welche von den Probanden/-innen als bedeutend angesehen wurden. Viele der von Walsh et al. (2003) aufgeführten Punkte können unter dem Überbegriff funktionelle Beeinträchtigung (,Disability“) zusammengefasst werden. Kamper et al. (2014) verweisen darauf, dass der Roland Morris Disability Questionnaire (RMDQ) zwar ein valides Instrument für die Messung von funktioneller Beeinträchtigung sei, jedoch nicht alle individuellen funktionellen Probleme der Betroffenen berücksichtigen kann.
So zeigen auch Eaves et al. (2015), dass Patienten/-innen sich andere Ergebnisse einer Behandlung vorstellen, die jedoch teilweise auf Schmerzreduktion und einer Verbesserung der funktionellen Beeinträchtigung basieren. Patienten/-innen wünschten sich eine langfristige Kontrolle des Schmerzes und adäquate Bewältigungsstrategien sowie langfristig eine Steigerung der Lebensqualität und des allgemeinen Wohlbefindens (Eaves et al., 2015, S. 4ff). Zusätzlich wird eine größere Akzeptanz von chronischen Rückenschmerzen in der Gesellschaft gewünscht.

\section{Folgen für die Gesellschaft}

Die Folgen von chronischen Rückenschmerzen sind in Deutschland gravierend. Laut Gesundheitsberichterstattung des Bundes betrugen 2006 die Kosten für die Versorgung von Patienten/-innen mit unspezifischen Rückenschmerzen 8,4 Milliarden Euro pro Jahr, wobei $85 \%$ auf die Arbeits- und Erwerbsunfähigkeit entfielen und $15 \%$ auf die medizinische Behandlung (BÄK et al., 2010, S. 42).

Die Gesamtheit der Arbeitsunfähigkeitstage (AU-Tage) pro Versicherungsjahr aufgrund von „Krankheiten des Muskel-Skelett-Systems“ belaufen sich im Jahr 2013 bei der Deutschen Angestellten Versicherung auf 313 (DAK, 2014, S. VI), bei der Techniker Krankenkasse sind es im Jahr 2012289 AU-Tage pro 100 Versichertenjahre (TK, 2013, S. 108). Anhand dieser Zahlen wird deutlich, dass die Folgen insbesondere aufgrund indirekter Kosten durch Arbeitsunfähigkeit und Rente enorm sind, dies spiegelt sich in der Zahl von 135.000 verlorenen Erwerbstätigkeitsjahren 2008 mit der Diagnose M54 „Rückenschmerzen“ (RKI, 2012, S. 16).

28,4\% aller Medikamente (Rheuma- und Schmerzmittel) wurden für muskuloskelettale Erkrankungen verordnet, ein Großteil davon für Rückenschmerzen (BKK, S. 24f.).

\section{Die derzeitige Versorgung durch die Gesundheitsfachberufe}

Neben der medikamentösen Therapie stehen bei chronischem Rückenschmerz weitere nichtmedikamentöse Behandlungsoptionen zur Verfügung. Die AWMF-Leitlinie (AWMF, 2016) empfiehlt als sinnvolle Maßnahmen zur Behandlung chronischer Rückenschmerzen Bewegungstherapie, Schulungsmaßnahmen (Beratung/Edukation) und kognitive Verhaltenstherapie (KVT), eingebunden in ein multimodales Behandlungskonzept. Ergänzend können Entspannungsverfahren, ergotherapeutische Maßnahmen im Rahmen multimodaler Programme und Rückenschule eingesetzt werden. Optional angeboten werden können Akupunktur, Manipulation/Mobilisation sowie Massage in Kombination mit Bewegungstherapie. 
Nicht empfohlene Maßnahmen laut AWMF (2016) sind Bettruhe, Massage oder invasive Therapieverfahren (perkutan oder operativ).

Bei weiterhin bestehenden Risikofaktoren, nachgewiesenen psychosozialen Folgen oder symptomunterhaltenden Nebenerkrankungen wie Angst oder Depression sind nach der Entlassung aus der schmerztherapeutischen Behandlung oder Rehabilitation besondere Maßnahmen erforderlich. (RKI, 2012, S. 22ff.)

Für die bundesweite Versorgung existieren keine präzisen Angaben dazu, wiehäufig PhysiotherapieundErgotherapie für die betroffenen Patienten verordnet wurde. Für den Heilmittelbericht 2014 hat das Wissenschaftliche Institut der AOK (WIdO) die im Jahr 2013 für etwa 70 Millionen GKV-Versicherte ausgestellten Heilmittelrezepte analysiert. Fast jeder zweite Besuch $(48,8 \%)$ in der physiotherapeutischen Praxis diente der Behandlung von Rückenbeschwerden. Keine näheren Angaben ließen sich zu den Verordnungen für Physiotherapie in der PKV finden.

Die in den AWMF-Leitlinie empfohlenen Therapieformen sollten auf der Grundlage eines strukturierten interdisziplinären Assessments inhaltlich und zeitlich sowie auf die individuellen Bedürfnisse der Betroffenen abgestimmt werden. Dies basiert auf der Annahme, dass chronischer Rückenschmerz physische, psychologische und soziale Beeinträchtigungen einschließt. Folglich sollten bei einer multimodalen Therapie die verschiedenen biopsychosozialen Komponenten gleichzeitig berücksichtigt werden. Dies sind medizinische (Pharmakotherapie, Edukation), physische (Bewegungstherapie), berufsbezogene und verhaltenstherapeutische Komponenten und sollten von Angehörigen mindestens dreier Berufsgruppen mit unterschiedlichem therapeutischem Hintergrund durchgeführt werden, z. B. aus Medizin, Physiotherapie, Sporttherapie, Psychotherapie oder Ergotherapie (AWMF, 2016).

Von Personen mit chronischen Rückenschmerzen erhielten $8 \%$ Rehabilitationsleistungen (RKI, 2012, S. 19). Die Rehabilitation erfolgt teilweise bereits in Form von multimodalen Programmen (s. a. Abschnitt 5). Der Anteil ambulanter Rehabilitationen erhöht sich dabei stetig. Er lag 2010 bei $22 \%$ und bei den Anschlussheilbehandlungen bei $32 \%$.

Obwohl ambulant und stationär Patienten/-innen mit Rückenschmerzen multimodal behandelt werden, sind die Kriterien oder Leitlinien, nach denen die multimodalen Therapien verordnet werden, wenig bekannt. In der Regelversorgung kommt es zu Überund Unterversorgungen sowie Fehlversorgungen bei der derzeitigenDiagnostikundTherapievonRückenschmerzen beispielsweise durch den nicht adäquaten Einsatz bildgebender Verfahren oder therapeutischer Injektionen
(RKI, 2012, S.22). Die Unterversorgung betrifft vor allem die mangelnde medizinische und berufliche Rehabilitation von Personen mit schweren chronisch beeinträchtigenden Rückenleiden.

Weiterhin zeigt sich immer noch ein steigender Einsatz von Schmerzmitteln, spinalen Injektionen, Bandscheibenoperationen und Wirbelversteifungen in der Versorgung der Betroffenen, obwohl der Nutzen dieser Therapien nicht erwiesen ist (Deyo, Mirza, Turner \& Martin, 2009, S. 62ff.). Aufgrund der unbefriedigenden postoperativen Ergebnisse scheint eine zurückhaltende Indikationsstellung für Operationen empfehlenswert (RKI, 2012, S. 24).

Im internationalen Vergleich schneidet Deutschland bei den Rückkehrraten zur Arbeit von Personen mit Arbeitsunfähigkeit bedingt durch chronischen Rückenschmerz schlechter ab. Es nahmen nur $54 \%$ nach zwei Jahren ihre Arbeit wieder auf, in Holland waren es $81 \%$. Gründe könnten eine unscharfe Auswahl der Versicherten (ICD-10-Klassifikation), ihre unzureichende und einseitige Behandlung, die häufige fehlende Nachsorge und/oder eine ungenügende Berücksichtigung beruflicher Belastungen sein (RKI, 2012, S. 24).

\section{METHODE}

Für die Informationen zu dem Gesundheitsproblem wurde eine Recherche im Januar und Februar 2015 sowie im November 2016 in PubMed mit den Suchworten ,,chronic low back pain“, „multidisciplinary biopsychosocial rehabilitation“, „patient centered outcomes“ durchgeführt. Bei der Suche nach qualitätsgesicherten Reviews wurde ein relevantes Cochrane Review gefunden mit den Suchbegriffen „physiotherapy“ AND „occupational therapy“ AND „chronic low back pain“, mit Suchfilter „Cochrane Reviews“. Zur Ergänzung des Cochrane Reviews konnten keine weiteren relevanten randomisierten kontrollierten Studien (RCT) gefunden werden.

Informationen zur derzeitigen Versorgung durch den entsprechenden Gesundheitsfachberuflieferten Statistiken der Gesundheitsberichterstattung des Bundes (Robert Koch Institut) und des Wissenschaftlichen Instituts der AOK sowie Gesundheitsreporte der DAK und TK ebenso wie der Heil- und Hilfsmittelreport der Barmer GEK. Hinweise zu klienten/-innenzentrierten Endpunkten wurde mit den Stichworten „klientenzentrierte Endpunkte“, „patientenzentrierte Endpunkte“, „patient centered outcomes“ AND „chronic low back pain“ bei Google Scholar, Pubmed und dem PCORI (PatientCentered Outcomes Research Institute) gesucht.

Informationen zur Intervention, die zur Lösung des Gesundheitsproblems beitragen könnten, inklusive potentiellem Wirkmechanismus fanden sich im 
Heilmittelkatalog und der Leitlinie Kreuzschmerz. Zudem wurden mit den Stichworten „Manuelle Therapie“, „Physiotherapie“, „Übungstherapie“, „medizinische Therapie“, „Ergotherapie“, „Psychotherapie“, „manual therapy“, „physiotherapy“, ,physical therapy“, ,,exercise therapy“, „occupational therapiy“, „medical therapy“, „psychotherapy“, ,intervention“ AND „,chronic low back pain" in der Cochrane Datenbank, Google Scholar sowie in Pubmed Studien zu der Evidenzlage von Monotherapien herausgesucht.

Die Bewertung der Evidenzqualität erfolgte nach den Empfehlungen der GRADE Arbeitsgruppe (Meerpohl et al., 2012).

\section{EVIDENZLAGE}

Beschreibung der Intervention multidisziplinärer biopsychosozialer Rehabilitation (MBR) und deren Wirkmechanismen auf klienten/-innenzentrierten Endpunkte

Wissenschaftliche und wirtschaftliche Begründungen der Behandlung von chronischen Rückenschmerzen beispielsweise in Form von Leitlinien können helfen, die Versorgung dieser Patienten/-innen effektiver und kostengünstiger zu gestalten (RKI, 2012, S. 23). Patienten/-innen mit chronischem unspezifischem Rückenschmerz sollen der AWMF Leitlinie zufolge, wenn andere sinnvolle Therapieverfahren in Kuration oder Rehabilitation unzureichend wirksam waren, mit multimodalen Programmen behandelt werden (AWMF, 2016).

$\mathrm{Zu}$ den Zielen der multimodalen Behandlung gehören unter anderem die Reduktion von Schmerzen und schmerzbedingter Beeinträchtigung, die Verbesserung von Ausdauer, Muskelkraft, Koordination und Beweglichkeit und die Verbesserung der funktionellen Leistungsfähigkeit. Des Weiteren beinhaltet sie die Verminderung psychosozialer Belastungen und psychischer Folgen oder Komorbiditäten und den Abbau inadäquater Bewältigungsstrategien. Die Motivierung sowie die Verbesserung der psychischen und sozialen Kompetenzen in Alltag und Beruf sind zentrale Bestandteile. Berufliche (Wieder-) Eingliederung bei unspezifischem Rückenschmerz ist ein wesentliches Behandlungsziel (AWMF, 2016)

Hintergrund ist die Evidenzlage verschiedener Monotherapien wie Bewegungsübungen, Manuelle Therapie und Training der stabilisierenden Muskulatur, die meist nur geringe Wirksamkeit zeigten (Ferreira et al., 2007, S. 31ff.). Auch Trainingstherapie schneidet nicht besser als andere konservative Behandlungen ab (Hayden, van Tulder, Malmivaara \& Koes, 2005, S. 8f.; Unsgaard-Tondel, Fladmark, Salvesen \& Vasseljen, 2010, S. 1426-1440).
Positive Ergebnisse zeigten sich bei Interventionen, die die beitragenden psychologischen Faktoren wie Stress berücksichtigen und Selbstmanagement in der Schmerzkontrolle sowie die Entwicklung von Copingstrategien fokussieren (Woby, Roach, Urmston \& Watson, 2007, S. 869ff.). Beispielsweise ist das Konzept der „gelben Flaggen“ zur Früherkennung psychosozialer Risikofaktoren geeignet und liefert Hinweise auf ein erhöhtes Chronifizierungsrisiko (RKI, 2012, S. 22). Diese Ansätze fließen in die MBR ein, welche bessere Ergebnisse bei Patienten/-innen mit chronischen Rückenschmerzen erwarten lassen als Monotherapien (Kamper et al., 2014, S. 6). Um die in der Rehabilitation erreichten positiven Veränderungen bei chronischem Rückenschmerz beizubehalten, sollte ein ausgearbeiteter Nachsorgeplan mit der Entlassung aus der Klinik oder dem ambulanten Programm vorliegen und im Alltag sowie in der Arbeitswelt ansetzen (RKI, 2012, S. 23).

\section{Endpunktspezifische Zusammenfassung der Evidenzlage und Identifizierung der Evidenzlücken}

Zur Ermittlung der Evidenzlage wurde ein aktuelles Cochrane Review (Kamper et al., 2014) in Bezug auf die Langzeitwirkung der MBR analysiert. Insgesamt 41 RCTs wurden in das Review eingeschlossen, sieben davon bezogen sich auf die Endpunkte funktionelle Beeinträchtigung und Schmerz, die auf Grundlage der Ergebnisse aus Abschnitt 2 als wichtigste patienten/innen zentrierte Endpunkte ausgewählt wurden.

Sechs Studien mit 722 erwachsenen Personen untersuchten den Endpunkt funktionelle Beeinträchtigung. Vier Studien wurden in Europa (Niederlanden, Schweden, Norwegen und Finnland), eine im Iran und eine in den USA durchgeführt.

SiebenStudienmit821 erwachsenenPersonenuntersuchten den Endpunkt Schmerz. Es handelt sich um die gleichen Studien, wie für den Endpunkt Beeinträchtigung und um eine zusätzliche Studie (Bendix, A., Bendix, T., Haestrup \& Busch, 1998) aus Dänemark. Daher werden beide Endpunkte zusammen betrachtet und die Abweichungen für Schmerz hervorgehoben.

Die Dauer der Behandlungen variiert von sechs (Von Korff et al., 2005) bis zu 135 Stunden (Bendix et al. 1996; Bendix et al., 1998) über einen Behandlungszeitraum von vier Terminen (Von Korff et al., 2005) bis zu zwölf Wochen (Lambeek, van Mechelen, Knol, Loisel \& Anema, 2010). Die Behandlungen wurden in ambulanten und stationären Settings durchgeführt. Die multidisziplinären Interventionen unterschieden sich sowohl im Inhalt, der Form, den beteiligten Berufsgruppen, den Voraussetzungen der Patienten/innen wie auch der Einbeziehung der Partner/-innen oder Arbeitgeber/-innen. Bei Lukinmaa (1989) wird die MBRIntervention nicht genauer beschrieben. Im Rahmen 
der MBR-Interventionen wird nur in zwei Studien (Bendix et al., 1996; Bendix et al., 1998; Linton, Boersma, Jansson, Svärd \& Botvalde, 2005) von Schmerzedukation oder einem Training des Copings berichtet.

Die Kontrollintervention (,usual care“) war nur unzureichend als „normale“ Versorgung durch medizinische Spezialisten in den Niederlanden (Lambeek et al., 2010), ,normale“ Versorgung in Dänemark (Bendix et al., 1996; Bendix et al., 1998), „,normale“ Versorgung in der Gesellschaft und gewöhnliche Medikation (Von Korff et al., 2005) sowie als orthopädische ambulante Intervention nach biomedizinischem Konzept (Lukinmaa, 1989) beschrieben.

Bei der Messung des Endpunktes funktionelle Beeinträchtigung (,Disability“) wurde bei fünf von sechs Studien der Roland Morris Disability Questionnaire (RMDQ) verwendet, nur in einer Studie (Strand, Ljunggren, Haldorsen \& Espehaug, 2001) wurde auch die Rückkehr zur Arbeit („Return to Work“) gemessen. Für den Endpunkt Schmerz verwenden sechs von sieben Studien gut vergleichbare Messinstrumente (VAS und NRS). Der Messzeitpunkt für das Follow-up ist bei beiden Endpunkten zwölf Monate nach Interventionsbeginn, nur in zwei Studien (Bendix et al. 1996; 1998) wurde der Endpunkt Schmerz schon nach vier Monaten gemessen. Für beide Endpunkte liegt ein gepolter Effektschätzer vor (SMD Schmerz -0,21 [CI 95\% -0,37; -0,04] und Beeinträchtigung $-0,23$ [CI $95 \%-0,4 ;-0,06]$ ), welcher kleine, jedoch signifikante und klinisch relevante Effekte zeigt.

Die Qualität der eingeschlossenen Studien ist unterschiedlich. Eine Verblindung der Probanden/-innen und Untersucher/-innen war bei keiner Studie gegeben. Auch die Kontrollinterventionen waren nicht ausreichend beschrieben. Bei Lambeek et al. (2010) werden Teile der Untersuchung nicht im Ergebnisteil dargestellt. Die Studien von Lukinmaa (1989) und Von Korff et al. (2005) haben eine lückenhafte Darstellung der Randomisierung, der Kointerventionen und der Ergebnisse.

Evidenzlücken bestehen in Bezug auf die Konzeption der Interventionen, der Kontrollintervention, die benötigte Dauer und Frequenz der Interventionen und für die klienten/-innenrelevanten Endpunkte. Auch die Kosten für die MBR sind bisher unzureichend untersucht worden.

\section{Übertragbarkeit der Intervention in die Versorgung}

Im Folgenden wird die Übertragung der Ergebnisse des Reviews (Kamperetal., 2014) auf den Versorgungskontext in Deutschland anhand des PICO-Schemas dargestellt.

Population: Es wurden alle Studien berücksichtigt, die volljährige Probanden/-innen mit einem anhaltenden Rückenschmerz von länger als drei Monaten einschlossen.
Dabei wurde hinsichtlich der Lokalisation darauf Wert gelegt, dass mindestens $75 \%$ der Probanden/-innen einer Studie Schmerzen im Bereich der Lendenwirbelsäule angaben. Als Ausschlusskriterium wurden spezifische Ursachen wie beispielsweise Frakturen oder Infektionen benannt (Kamper et al., 2014, S. 7)

Intervention: Die Interventionen der einzelnen Studien waren sowohl in ihrer Dauer als auch in ihrer inhaltlichen Beschreibung sehr heterogen. Ebenso konnte aus der Übersicht der Studien nicht entnommen werden, ob ein Manual oder andere standardisierte Instruktionen zugrunde lagen (Kamper et al., 2014). Hinsichtlich der Intervention sollte eine gute Nachvollziehbarkeit sichergestellt werden. Für die Übertragung in den deutschen Kontext gibt es jedoch bereits Vorlagen, wie beispielsweise das „GRIP-Manual“. Zusätzlich zu einer klaren Struktur, würde dieses Manual den Vorteil der weiteren Individualisierung der Intervention mit sich bringen. Durch eine therapeutische Schwerpunktsetzung auf Basis der Eingangsuntersuchungen kann eine manualkonforme, individuelle Anpassung derBehandlung erfolgen. (Hildebrandt et al., 1996, S. 190-203)

Control: Die aktuelle Versorgungssituation in Deutschland wurde bereits in Abschnitt 4 kurz dargestellt. Da die Kontrollinterventionen der einbezogenen Studien nicht näher beschrieben werden, ist die Übertragbarkeit nur schwer zu überprüfen.

Outcome: Die primären Endpunkte des Reviews sind Schmerz und Beeinträchtigung. Diese stimmen teilweise mit den in Abschnitt 2 dargestellten klienten/innenzentrierten Endpunkten überein. Es muss weiterhin überlegt werden, ob diese allgemein gehaltenen Endpunkte genauer aufgegliedert werden sollten. Der relativ globale Begriff funktionelle Beeinträchtigung (Disability) könnte zuunterschiedlichinterpretiertwerden.EineSpezifizierung in patienten/-innen relevante Aktivitäten wie von Walsh et al. (2003, S. 91f.) empfohlen, stellt jedoch höhere methodische Ansprüche. Hier wäre der Einsatz von patienten/-innenspezifischen Messinstrumenten wie dem PSFS (Heldmann, Schöttker-Königer \& Schäfer, 2015) $\mathrm{zu}$ empfehlen. Ferner sollte ein Kompromiss zwischen klienten/-innen- und gesellschaftszentrierten Endpunkten gefunden werden (Walsh et al., 2003, S. 91f.).

\section{FORSCHUNGSEMPFEHLUNG}

\section{Begründete Empfehlung für eine Mach- barkeitsstudie}

AufBasis der vorhandenen Evidenzlage empfiehlt sicheine Machbarkeitsstudie zur manualisierten und individuell angepassten MBR im Vergleich zur Routineversorgung bei Patienten/-innen mit chronischem Rückenschmerz im erwerbsfähigen Alter in Deutschland. Die deutsche 
Rentenversicherung umfasst damit alle Personen zwischen 15 und 65 Jahren (RKI, 2012, S. 16). Für diese Altersgruppe erscheint die Relevanz der angemessenen Therapie am höchsten, da es durch chronische Rückenschmerzen zu physischen und psychischen Beeinträchtigungen, Arbeitsunfähigkeit und damit zu einer familiären und finanziellen Belastung kommen kann. Auch für die Gesellschaft und die Kostenträger ist die Bedeutung aufgrund der finanziellen Belastung durch Arbeitsunfähigkeit und drohender Berentung hoch. Im Koalitionsvertrag der Bundesregierung ist festgehalten, dass die strukturierten Behandlungsprogramme für chronisch Kranke weiterentwickelt und dass neue Programme für die Behandlung von Rückenleiden entwickelt werden sollen (Deutscher Bundestag, 2013, S. 77),

Als Vorbereitung für eine entsprechende Wirksamkeitsstudie ist eine Machbarkeitsstudie notwendig. Es gilt, zuerst den Zugang zur Population $\mathrm{zu}$ überprüfen und ein geeignetes Setting zu eruieren. Anschließend ist eine Recherche zu den Versorgungsdaten mit MBR durchzuführen. Vorhandene Manuale wie beispielsweise das GRIP-Manual müssen auf ihre Anwendbarkeit als Behandlungsprogramm geprüft werden. Dieses basiert auf einem Entwurf aus den USA, scheint jedoch auf Deutschland übertragbar zu sein, da es bereits teilweise in modifizierter Form verwendet wird. Es beinhaltet ausführliche Informationen über die Ziele der Behandlung, Trainings- und Übungstherapie, verhaltenstherapeutische Behandlungsmaßnahmen zur Veränderung von maladaptiven, auf Ruhe und Schonung ausgerichteten Krankheitsverhalten sowie ergotherapeutischen Maßnahmen. Das GRIP-Programm berücksichtigt individuelle Arbeitsplatzanforderungen und zielt auf Rückkehr zur Arbeit oder körperliche Aktivität ab (Hildebrandt et al., 1996, S. 192-193).

Ein weiterer wichtiger Aspekt ist die Validität der Assessments, um eine adäquate Gewichtung der Therapiezusammensetzung im Rahmen der MBR gestalten zu können. Auf Basis der Evidenzlage sind die Endpunkte funktionelle Beeinträchtigung, Rückkehr zur Arbeit und Schmerz zu wählen. Dem Endpunkt Rückkehr zur Arbeit wurde seitens der Klienten/-innen zwar recht geringe Bedeutung zugeschrieben, er spielt jedoch für Kostenträger und Gesellschaft eine bedeutende Rolle und damit für die Finanzierung von solchen Therapiemodellen. $\mathrm{Zu}$ prüfen wäre auch, ob die Rückkehr zur Arbeit auch für die Patienten/-innen auf Grund positiver psychischer und sozialer Effekte langfristig eine größere Bedeutung bekommen könnte. Ein mögliches Assessment könnte hierfür das Canadian Occupational Performance Measure sein (Walsh et al., 2003).

Als Setting für die Studie wären Rehabilitationszentren geeignet, da diese zum einen multiprofessionelle Versorgung und zum anderen den Zugang zu Probanden mit chronischen Rückenschmerzen gewährleisten können. Hierfür müssen potentielle Partner ausfindig gemacht werden.

\section{Literaturverzeichnis}

AWMF (2016). Nationale Versorgungsleitlinie Nicht-spezifischer Kreuzschmerz - Konsultationsfassung. Berlin: BÄK, KBV, AWMF.

Bendix, A. E., Bendix, T., Haestrup, C., Busch, E. (1998). A prospective, randomized 5-year follow-up study of functional restoration in chronic low back pain patients. European Spine Journal, 7 (2), 111-9.

Bendix, A. F., Bendix, T., Vaegter, K., Lund, C., Frolund, L., Holm, L. (1996). Multidisciplinary intensive treatment for chronic low back pain: a randomized, prospective study. Clinical Journal of Medicine, 63, 62-9.

BKK Dachverband e.V. (Hrsg.) (2013). Gesundheit in Bewegung. Schwerpunkt Muskel- und Skeletterkrankungen. BKK Gesundheitsreport (2013). Berlin. http://www.bkk-dachverband. de/fileadmin/publikationen/gesundheitsreport_2013/BKKGesundheitsreport_2013.pdf. (Zugriff: 06.02.2015)

Borkan, J., Reis, S., Hermoni, D., Biderman, A. (1995). Talking about the pain: A patient-centered study of low back pain in primary care. Social Science \& Medicine, 40 (7), 977-988.

Bundesärztekammer (B̈̈K), Kassenärztliche Bundesvereinigung (KBV), Arbeitsgemeinschaft derWissenschaftlichen Medizinischen Fachgesellschaften(AWMF) (2010). Nationale Versorgungsleitlinie Kreuzschmerz - Langfassung. Version 4. Zuletzt verändert: August
2013. DOI: 10.6101/AZQ/000149. http://www.kreuzschmerz. versorgungsleitlinien.de. (Zugriff: 06.02.2015)

Bunzli, S., Watkins, R., Smith, A., Schütze, R., O'Sullivan, P. (2013). Lives on hold: A qualitative synthesis exploring the experience of chronic low back pain. The Clinic Journal of Pain 29 (10), 907-916.

Deutsche Angestellten Krankenkasse (DAK) (2014). Gesundheitsreport 2014. Die Rushhour des Lebens. Gesundheit im Spannungsfeld von Job, Karriere und Familie. www.dak.de/dak/download/ Vollstaendiger_bundesweiter_Gesundheitsreport_2014-1374196. pdf. (Zugriff: 06.02.2015)

Deutscher Bundestag (2013). Deutschlands Zukunft gestalten. Koalitionsvertrag zwischen CDU, CSU und SPD. 18. Legislaturperiode. http://www.bundesregierung.de/Content/ DE/_Anlagen/2013/2013-12-17-koalitionsvertrag.pdf;jsess ionid=2DCF1375B2D992D8D2502E19820C0FD3.s4t2?_blob=publicationFile \&v=2. (Zugriff: 20.02.2015)

Deyo, R. A., Mirza, S. K., Turner, J. A., Martin, B. I. (2009). Overtreating Chronic Back Pain: Time to Back Off? The Journal of the American Board of Family Medicine, 22 (1), 62-68.

Duthey, B. (2013). Background Paper 6.24 Low back pain. Retrieved from http://www.who.int/medicines/areas/priority_medicines/ BP6_24LBP.pdf 
Eaves, E. R., Sherman, K. J., Ritenbaugh, C., Hsu, C., Nichter, M., Turner, J. A., Cherkin, D. C. (2015). A qualitative study of changes in expectations over time among patients with chronic low back pain seeking four CAM therapies. BMC Complementary and Alternative Medicine 15 (12).

Ferrans, C. E., Zerwic, J. J., Wilbur, J. E. \& Larson, J. L. (2005). Conceptual model of health-related quality of life. Journal of nursing scholarship: an official publication of Sigma Theta Tau International Honor Society of Nursing / Sigma Theta Tau, 37(4), 336-342.

Ferreira, M. L., Ferreira, P. H., Latimer, J., Herbert, R. D., Hodges, P. W., Jennings, M. D., Maher, C., Refshauge, K. M. (2007). Comparison of general exercise, motor control exercise and spinal manipulative therapy for chronic low back pain: A randomized trial. Pain 131 (1-2), 31-37.

Hayden, J. A., van Tulder, M. W., Malmivaara, A. V., Koes, B. W. (2005). Meta-analysis: exercise therapy for nonspecific low back pain. Cochrane Database Systematic Reviews. www.ncbi.nlm.nih. gov/pubmed/16034851. (Zugriff: 24.02.2015)

Heilmittelkatalog Ergotherapie (2011). Heilmittelkatalog. Nachschlagewerk zur Ermittlung verordnungsfähiger Heilmittel entsprechend den Indikationen. Ludwigsburg: Intellimed. www.heilmittelkatalog.de/files/hmk/ergo/index.htm. (Zugriff: 06.02.2015)

Heilmittelkatalog Physikalische Therapie (2011). Heilmittelkatalog. Nachschlagewerk zur Ermittlung verordnungsfähiger Heilmittel entsprechend den Indikationen. Ludwigsburg: Intellimed. www. heilmittelkatalog.de/files/hmk/physio/index.htm. (Zugriff: 06.02.2015)

Heldmann, P., Schöttker-Königer, T. \& Schäfer, A. (2015). Crosscultural Adaptation and Validity of the "Patient Specific Functional Scale“. International Journal of Health Professions, 2(1), 73-82. doi:10.1515/ijhp-2015-0002

Hildebrandt, J., Pfingsten, M., Lüder, S., Lucan, S., Pauls, J., Seeger, D., Strube, J., von Westernhagen, S., Wendt, A. (Hrsg.) (2003) Göttinger Rücken Intensiv Programm (GRIP) - Das Manual Berlin: Congress Compact Verlag.

Hildebrandt, J., Pfingsten, M., Franz, C., Saur, P., Seeger, D. (1996). Das Göttinger Rücken Intensiv Programm (GRIP) - ein multimodales Behandlungsprogramm für Patienten mit chronischen Rückenschmerzen, Teil 1. Der Schmerz 10, 190-203.

Kamper, S., Apeldoorn, A., Chiarotto, A., Smeets, R., Ostelo, R., Guzman, J., van Tulder, M. (2014). Multidisciplinary biopsychosocial rehabilitation for chronic low back pain. Cochrane Database of Systematic Reviews (9). www.ncbi.nlm.nih.gov/ pubmed/25180773. (Zugriff: 24.02.2015)

Kohlmann, T. (2003). Die Chronifizierung von Rückenschmerzen. Ergebnisse eines internationalen WHO-Workshops. Bundesgesundheitsbildung. Gesundheitsforschung. Gesundheitsschutz 46, 327-335.

Lambeek, L. C., van Mechelen, W., Knol, D. L., Loisel, P., Anema, J. R. (2010). Randomised controlled trial of integrated care to reduce disability from chronic low back pain in working and private life. British Medical Journal, 340 (7749), 750
Langer, G., Meerpohl, J. J., Perleth, M., Gartlehner, G., KaminskiHartenthaler, A., \& Schunemann, H. (2012). [GRADE guidelines: 1. Introduction - GRADE evidence profiles and summary of findings tables]. Zeitschrift fur Evidenz, Fortbildung und Qualitat im Gesundheitswesen, 106(5), 357-368. doi:10.1016/j. zefq.2012.05.017

Linton, S. J., Boersma, K., Jansson, M., Svärd, L., Botvalde, M. (2005). The effects of cognitive-behavioral and physical therapy preventive interventions on pain-related sick leave: a randomized controlled trial. Clinical Journal of Pain, 21 (2), 109-119.

Lukinmaa, A. (1989) Low back pain as a biopsychosocial problem. A controlled clinical trial and a costeffectiveness analysis. Kansanelakelaitoksen Julkaisuja, 1-90.

Meerpohl, J. J., Langer, G., Perleth, M., Gartlehner, G., KaminskiHartenthaler, A. \& Schunemann, H. (2012). [GRADE guidelines: 4. Rating the quality of evidence - limitations of clinical trials (risk of bias)]. Zeitschrift für Evidenz, Fortbildung und Qualität im Gesundheitswesen, 106(6), 457-469. doi:10.1016/j. zefq.2012.06.014

Monticone, M., Ambrosini, E., Rocca, B., Magni, S., Brivio, F., Ferrante, S. (2014). A multidisciplinary rehabilitation programme improves disability, kinesiophobia and walking ability in subjects with chronic low back pain: results of a randomised controlled pilot study. European Spine Journal 23, 2105-2113.

Murad, M. H., Montori, V. M., Ioannidis, J. P., Jaeschke, R., Devereaux, P. J., Prasad, K., . . Guyatt, G. (2014). How to read a systematic review and meta-analysis and apply the results to patient care: users' guides to the medical literature. Jama, 312(2), 171-179. doi:10.1001/jama.2014.5559

Pieber, K., Herceg, M., Quittan, M., Csapo, R., Müller, R., Wiesinger, G. F. (2014). Long-term effects of an outpatient rehabilitation program in patients with chronic recurrent low back pain. European Spine Journal 23, 779-785.

Plass, D., Vos, T., Hornberg, C., Scheidt-Navas, C., Zeeb, H., Krämer, A. (2014). Trends in Disease Burden in Germany. Deutsches Ärzteblatt International, 111, 626-638.

Robert Koch Institut (2012). Gesundheitsberichterstattung des Bundes: Rückenschmerz. Heft 53. Berlin: RKI.

Statistisches Bundesamt (2012): https://www.destatis. de/DE/ZahlenFakten/Gesell-schaftStaat/Gesundheit/ VorsorgeRehabilitationseinrichtungen/Tabellen/Reha_ DiagnosenInsgesamt.html. (Zugriff: 03.02.2015)

Strand, L. I., Ljunggren, A. E., Haldorsen, E. M., Espehaug, B. (2001). The impact of physical function and pain on work status a 1-year follow-up in patients with back pain. Spine, 26 (7), 800-808.

Tavafian, S. S., Hamshidi, A. R., Mohammad, K. (2014). Treatment of low back pain: randomized clinical trial comparing a multidisciplinary group-based rehabilitation programme with oral drug treatment up to 12 months. International Journal of Rheumatic Disease 17, 159-164.

Techniker Krankenkasse (TK) (2013). Gesundheitsreport 2013. Berufstätigkeit, Ausbildung und Gesundheit, Band 28.

Unsgaard-Tøndel, M., Fladmark, A. M., Salvesen, Ø., Vasseljen, O. (2010). Motor control exercises, sling exercises, and general 
exercises for patients with chronic low back pain: a randomized controlled trial with 1-year follow-up. Physical Therapy. 90 (10), 1426-40.

Voigt-Radloff, S., Lang, B., \& Antes, G. (2014). [Potentials for research and innovations in allied health professions in Germany]. Zeitschrift für Evidenz, Fortbildung und Qualität im Gesundheitswesen, 108 Suppl 1, S4-8. doi:10.1016/j.zefq.2014.09.011

Voigt-Radloff, S., Stemmer, R., Behrens, J., Horbach, A., Ayerle, G. M., Schäfers, R., . . . Kempf, S. (2016). Forschung zu komplexen Interventionen in der Pflege- und Hebammenwissenschaft und in den Wissenschaften der Physiotherapie, Ergotherapie und Logopädie. Retrieved from Freiburg: https://freidok.uni-freiburg. de/data/10702 (Zugriff am 06.02.2015)

Von Korff, M., Balderson, B. H., Saunders, K., Miglioretti, D. L., Lin, E. H., Berry, S. (2005). A trial of an activating intervention for chronic back pain in primary care and physical therapy settings. Pain, 113 (3), 323-30.
Waddell, G. (2004). The Back Pain Revolution (2nd ed.). Edinburgh: Churchill Livingstone.

Walsh, A., Kelly, J., Johnson, S., Shanmugasundram, R., Bennetts, K. (2003). Performance Problems of Patients with Chronic Low-Back Pain and the Measurement of Patient-Centered Outcome. Spine 29 (1), 87-93.

WHO (2006). Gesundheit im Schlaglicht: Deutschland 2004. http:// www.euro.who.int/__data/assets/pdf_file/0010/103222/GER_ Highlights_ger.pdf?ua=1. (Zugriff: 06.02.2015)

WIP-Diskussionspapier 2014 (Hrsg.) (2014). Die Heilmittelversorgung der PKV- und GKV-Versicherten im Vergleich. http://www.wippkv.de/veroeffentlichungen.html. (Zugriff: 06.02.2015)

Woby, S.R., Roach, N.K., Urmston, M., Watson, P.J. (2007). The relation between cognitive factors and levels of pain and disability in chronic low back pain patients presenting for physiotherapy. European Journal of Pain 11 (8), 869-77. 